The Astrophysical Journal, 652:1112-1121, 2006 December 1

(C) 2006. The American Astronomical Society. All rights reserved. Printed in U.S.A.

\title{
THE RADIAL DISTRIBUTION OF THE INTERSTELLAR MEDIUM IN DISK GALAXIES: EVIDENCE FOR SECULAR EVOLUTION
}

\author{
Michael W. Regan, ${ }^{1}$ Michele D. Thornley, ${ }^{1,2}$ Stuart N. Vogel ${ }^{3}$ Kartik Sheth, ${ }^{4}$ Bruce T. Draine, ${ }^{5}$ \\ David J. Hollenbach, ${ }^{6}$ Martin Meyer, ${ }^{1}$ Daniel A. Dale, ${ }^{7}$ Charles W. Engelbracht, ${ }^{8}$ \\ Robert C. Kennicutt, ${ }^{8}$ Lee Armus, ${ }^{4}$ Brent Buckalew, ${ }^{4}$ Daniela Calzetti, ${ }^{1}$ \\ Karl D. Gordon, ${ }^{8}$ George Helou, ${ }^{9}$ Claus Leitherer, ${ }^{1}$ Sangeeta Malhotra, ${ }^{1}$ \\ Eric Murphy, ${ }^{10}$ George H. Rieke, ${ }^{8}$ Marcia J. Rieke, ${ }^{8}$ and J. D. Smith ${ }^{8}$ \\ Received 2005 December 21; accepted 2006 April 24
}

\begin{abstract}
One possible way for spiral galaxies to internally evolve would be for gas to flow to the center and form stars in a central disk (pseudo-bulge). If the inflow rate is faster than the rate of star formation, a central concentration of gas will form. In this paper we present radial profiles of stellar and $8 \mu \mathrm{m}$ emission from polycyclic aromatic hydrocarbons (PAHs) for 11 spiral galaxies to investigate whether the interstellar medium in these galaxies contains a central concentration above that expected from the exponential disk. In general, we find that the two-dimensional $\mathrm{CO}$ and PAH emission morphologies are similar, and that they exhibit similar radial profiles. We find that in 6 of the 11 galaxies there is a central excess in the $8 \mu \mathrm{m}$ and $\mathrm{CO}$ emission above the inward extrapolation of an exponential disk. In particular, all four barred galaxies in the sample have strong central excesses in both $8 \mu \mathrm{m}$ and $\mathrm{CO}$ emission. These correlations suggest that the excess seen in the $\mathrm{CO}$ profiles is, in general, not simply due to a radial increase in the $\mathrm{CO}$ emissivity. In the inner disk, the ratio of the stellar to the $8 \mu \mathrm{m}$ radial surface brightness is similar for 9 of the 11 galaxies, suggesting a physical connection between the average stellar surface brightness and the average gas surface brightness at a given radius. We also find that the ratio of the $\mathrm{CO}$ to $8 \mu \mathrm{m}$ PAH surface brightness is consistent over the sample, implying that the $8 \mu \mathrm{m}$ PAH surface brightness can be used as an approximate tracer of the interstellar medium.
\end{abstract}

Subject headings: dust, extinction - galaxies: individual (NGC 628, NGC 3351, NGC 3521, NGC 3627, NGC 4321, NGC 4736, NGC 4826, NGC 5055, NGC 5194, NGC 6946, NGC 7331) galaxies: ISM — galaxies: structure — infrared: galaxies

\section{INTRODUCTION}

While interactions, mergers, and accretion are clearly important in galaxy evolution, galaxies can also evolve significantly by internally driven processes that redistribute angular momentum using stellar bars, spiral arms, or triaxial halos. Such processes are known collectively as secular evolution (see the review by Kormendy \& Kennicutt [2004] and references therein). As galaxies evolve, gas loses angular momentum to the stars and falls to a smaller radius. The gas may then form new stars and create a region of enhanced stellar surface density in the center of the disk. This is one method of forming what is known as a pseudobulge (see references in Kormendy \& Kennicutt 2004). A pseudobulge is primarily distinguished from a true bulge by the fact that it has an exponential light profile rather than an $\exp \left(-\right.$ const $\left.r^{1 / 4}\right)$ light profile. A central excess of molecular gas is strong evidence

\footnotetext{
${ }^{1}$ Space Telescope Science Institute, 3700 San Martin Drive, Baltimore, MD 21218; mregan@stsci.edu.

2 Department of Physics and Astronomy, Bucknell University, Lewisburg, PA 17837.

3 Department of Astronomy, University of Maryland, College Park, MD 20742.

${ }^{4}$ Spitzer Science Center, California Institute of Technology, Mail Stop 2206, Pasadena, CA 91125.

${ }_{5}$ Princeton University Observatory, Princeton, NJ 08544-1001.

${ }^{6}$ NASA Ames Research Center, Mail Stop 245-3, Moffett Field, CA $94035-$ 1000 .

7 Department of Physics and Astronomy, University of Wyoming, Laramie, WY 82071

8 Steward Observatory, University of Arizona, Tucson, AZ 85721.

9 California Institute of Technology, Mail Stop 314-6, Pasadena, CA 91101.

${ }^{10}$ Department of Astronomy, Yale University, P.O. Box 20801, New Haven, CT 06520 .
}

that gas is forming a pseudo-bulge and thus serves as a marker for current secular evolution. It is the molecular interstellar medium (ISM) that leads to star formation and is thus - rather than the atomic or ionized phase - the key to the formation of a pseudo-bulge.

Regan et al. (2001) examined the central distribution of molecular gas in 15 galaxies from the Berkeley-Illinois-Maryland Association Survey of Nearby Galaxies (BIMA SONG), a millimeter-wave CO survey of nearby spiral galaxies. In that paper, a central excess of $\mathrm{CO}$ emission above the inward extrapolation of an exponential disk was seen in 9 out of 15 galaxies. In a study of CO emission in 20 galaxies, Sakamoto et al. (1999) noted an enhancement in the central concentration of CO emission in barred spirals; the concentration was measured from a ratio of the average gas surface density in the central kiloparsec to that in the galactic disk. Recently, using data from BIMA SONG (Regan et al. 2001; Helfer et al. 2003) and other existing data, Sheth et al. (2005) studied the molecular gas distribution in a sample of 50 nearby spiral galaxies. They not only confirmed the enhanced central $\mathrm{CO}$ concentrations among the CO-bright barred spirals, but also showed that enhanced central CO concentrations are present among barred spirals in the CO-faint galaxies. Moreover, Sheth et al. (2005) found that the effect of bars in transporting molecular gas to the center is equally pronounced in late and early Hubble-type barred spirals, although there are cases where some early-type barred spirals have little or no gas within the bar, consistent with higher accretion rates and starbursts in these galaxies.

Although these $\mathrm{CO}$ studies provide good evidence for central molecular gas accretion, the observed excess could be due to a radial variation in the ratio of the CO luminosity and the mass of 
TABLE 1

Galaxy Parameters

\begin{tabular}{|c|c|c|c|c|c|c|c|c|}
\hline Galaxy & R.A. (J2000.0) & Decl. (J2000.0) & $\begin{array}{c}V_{\mathrm{LSR}} \\
\left(\mathrm{km} \mathrm{s}^{-1}\right)\end{array}$ & $\begin{array}{c}i \\
(\mathrm{deg})\end{array}$ & $\begin{array}{l}\text { P.A. } \\
\text { (deg) }\end{array}$ & Type & $\begin{array}{c}d \\
(\mathrm{Mpc})\end{array}$ & Distance Reference \\
\hline NGC $0628 \ldots \ldots \ldots \ldots . . . . . .$. & 013641.70 & +154659.4 & 657 & 24 & 25 & $\mathrm{SA}(\mathrm{s}) \mathrm{c}$ & 7.3 & 1 \\
\hline NGC $3351 \ldots \ldots \ldots \ldots . . . . . . .$. & 104357.98 & +114214.4 & 778 & 40 & 13 & $\mathrm{SB}(\mathrm{r}) \mathrm{b}, \mathrm{H}$ II & 10.1 & 2 \\
\hline NGC $3521 \ldots \ldots \ldots \ldots . . . . . .$. & 110549.26 & $\begin{array}{lll}-00 & 02 & 02.3\end{array}$ & 805 & 58 & 164 & SAB(rs)bc, LINER & 7.2 & 1 \\
\hline NGC $3627 \ldots \ldots \ldots \ldots . . . . . . .$. & 112015.07 & +125921.7 & 727 & 63 & 176 & $\mathrm{SAB}(\mathrm{s}) \mathrm{b}, \mathrm{Sy}$ & 11.1 & 3 \\
\hline NGC $4321 \ldots \ldots \ldots \ldots . . . . . . . .$. & 122254.84 & +154920.0 & 1571 & 30 & 154 & $\mathrm{SAB}(\mathrm{s}) \mathrm{bc}, \mathrm{H}$ II & 16.1 & 4 \\
\hline NGC $4736 \ldots \ldots \ldots \ldots . . . . . . .$. & 125053.06 & +410713.6 & 308 & 35 & 100 & (R)SA(r)ab, LINER & 4.3 & 1 \\
\hline NGC $4826 \ldots \ldots \ldots \ldots . . . . . . .$. & 125644.24 & +214105.1 & 408 & 54 & 111 & (R)SA(rs)ab, Sy & 4.1 & 1 \\
\hline NGC $5055 \ldots \ldots \ldots \ldots . . . . .$. & 131549.25 & +420149.3 & 504 & 56 & 101 & $\mathrm{SA}(\mathrm{rs}) \mathrm{bc}, \mathrm{H}$ II/LINER & 7.2 & 1 \\
\hline NGC $5194 \ldots \ldots \ldots \ldots \ldots . . . . . . .$. & 132952.35 & +471153.8 & 463 & 15 & 0 & SA(s)bc-pec, Sy 2.5 & 8.4 & 1 \\
\hline NGC $6946 \ldots \ldots \ldots \ldots . . . . . . . .$. & 203452.33 & +600914.2 & 48 & 54 & 65 & $\mathrm{SAB}(\mathrm{rs}) \mathrm{cd}, \mathrm{H}$ II & 5.5 & 1 \\
\hline NGC $7331 \ldots \ldots \ldots \ldots . . . . .$. & 223704.09 & +342456.3 & 821 & 62 & 172 & $\mathrm{SA}(\mathrm{s}) \mathrm{b}, \mathrm{LINER}$ & 15.1 & 5 \\
\hline
\end{tabular}

NoтE.-Units of right ascension are hours, minutes, and seconds, and units of declination are degrees, arcminutes, and arcseconds.

REFERENCES.- (1) Tully 1988; (2) Graham et al. 1997; (3) Saha et al. 1999; (4) Ferrarese et al. 1996; (5) Hughes et al. 1998.

the $\mathrm{H}_{2}$ gas. In this case, a central concentration of $\mathrm{CO}$ emission would not necessarily indicate the presence of more gas. Another caveat of note for the $\mathrm{CO}$ studies is a poor understanding of the distribution of disk emission due to the limited extent $\left(r \sim 100^{\prime \prime}\right)$ or sparse coverage of the observations (e.g., discrete pointings along the major and minor axis; Young et al. 1995). This leads to additional uncertainties in assessing the scale length of the exponential disk and the subsequent measurement of the average disk gas surface density.

Regan et al. (2001) found that the surface brightness of the stellar disk and that of the $\mathrm{CO}$ disk are correlated. The radial profiles of the $\mathrm{CO}$ and the near-infrared $K$-band emission show a similar ratio of surface brightnesses over a range of radii for most of the galaxies in the sample. This correlation implies that there is feedback between the stellar and molecular gas disks in these galaxies, but a measure of the shape of the radial profile over a wider range of radii is needed to confirm the correlation.

With the launch of the Spitzer Space Telescope, a new tracer of the ISM is available: emission from PAHs (see, for example, Helou et al. 2000; Pahre et al. 2004) at $8 \mu \mathrm{m}$. PAHs are large molecules or very small dust grains that are stochastically heated by optical and UV light and dominate the emission in the $8 \mu \mathrm{m}$ filter of the Infrared Array Camera (IRAC; Fazio et al. 2004) from spiral galaxies. Although the total PAH emission from a galaxy is correlated with the total amount of star formation in the galaxy (Roussel et al. 2001), this does not require that PAH emission be a tracer of star formation. In particular, Infrared Space Observatory (ISO) and submillimeter observations (Haas et al. 2002), as well as Spitzer observations (M. Meyer et al. 2006, in preparation), suggest that PAH emission is likely a better tracer of the dust content of the galaxy than the amount of star formation.

In this paper we look at 11 spiral galaxies that are common to the Regan et al. (2001) sample and the Spitzer Infrared Nearby Galaxies Survey (SINGS). SINGS is a Spitzer Legacy project to observe 75 nearby galaxies, using all three instruments of Spitzer, to investigate the relationship between star formation and the ISM in galaxies (Kennicutt et al. 2003). For the 11 galaxies in common, we compare the radial distribution of the $3.6 \mu \mathrm{m}$ IRAC images, which primarily trace the stellar component in the galaxy, to the $8 \mu \mathrm{m}$ PAH images and the BIMA SONG CO $2.6 \mathrm{~mm} \mathrm{CO}$ $(J=1-0)$ images, both of which trace the ISM in the galaxy. We find that the images of $8 \mu \mathrm{m}$ diffuse emission are highly correlated with the $\mathrm{CO}$ images, providing more evidence that the PAH emission is a good tracer of the ISM. We also find that in 6 out of 11 galaxies there is a central excess in the PAH emission, suggesting that the gas is being driven inward faster than it is forming stars. This net inflow of gas into the central region is an expectation of secular evolution.

\section{OBSERVATIONS AND DATA REDUCTION}

The 11 galaxies listed in Table 1 were observed with the IRAC between 2003 December 3 and 2004 July 28 . We observed all of the galaxies in the standard SINGS observing mode, which yields a total integration time of $240 \mathrm{~s}$ in the core of the mosaic and $120 \mathrm{~s}$ in the outer 2.5 minutes of the mosaic. The basic calibrated data (BCD) images of individual fields were then processed using the standard SINGS IRAC pipeline (Regan et al. 2004) to create the final mosaics. The primary added value of the SINGS pipeline is that it solves for both the best position and bias offset for each frame in the mosaic. It performs a cross correlation for each pair of overlapping images and then performs a $\chi^{2}$ minimization to find the optimum location. The residuals in the locations are around $1 / 10$ of an IRAC pixel. Once we have the correct relative location, we determine the correct DC level for each image by finding the difference in the pixels with fluxes in the lowest 20th percentile in the region of overlap between pairs of images and then performing a $\chi^{2}$ minimization. We use the 20th percentile pixels to minimize the effect of the "mux bleed" detector artifact. This artifact creates a positive bias to pixels in the fast-scan direction adjacent to a bright region. By using the 20th percentile pixel intensities, we reduce the residuals in the background matching by up to a factor of 3 compared to using the 50th percentile pixel intensities (the median). We then assemble the final mosaics with cosmic-ray rejection using the drizzle routine (Fruchter \& Hook 2002).

Although the $8 \mu \mathrm{m}$ emission that IRAC observes in channel 4 is primarily due to emission from PAHs, there is also a contribution from stellar photospheres (Helou et al. 2000). Meanwhile, the 3.6 and $4.5 \mu \mathrm{m}$ IRAC images are almost completely dominated by stellar photospheric emission. We created images of $8 \mu \mathrm{m}$ PAH emission using the method of Helou et al. (2004): $I_{\nu}(8 \mu \mathrm{mPAH})=I_{\nu}(8 \mu \mathrm{m})-0.232 I_{\nu}(3.6 \mu \mathrm{m})$. Note that throughout this paper we make no aperture corrections to the fluxes in either the 3.6 or $8 \mu \mathrm{m}$ images. Therefore, due to known calibration issues with the BCD images, the fluxes could be up to $20 \%$ too high at $8 \mu \mathrm{m}$. We show the final $3.6 \mu \mathrm{m}, 8 \mu \mathrm{m} \mathrm{PAH}$, and $\mathrm{CO}$ images in Figure 1. The angular resolution is approximately $2^{\prime \prime}$ in the IRAC images and $6^{\prime \prime}$ in the BIMA CO images.

To be able to obtain accurate radial profiles, we need to subtract the sky level. To determine the sky level in our images, we 

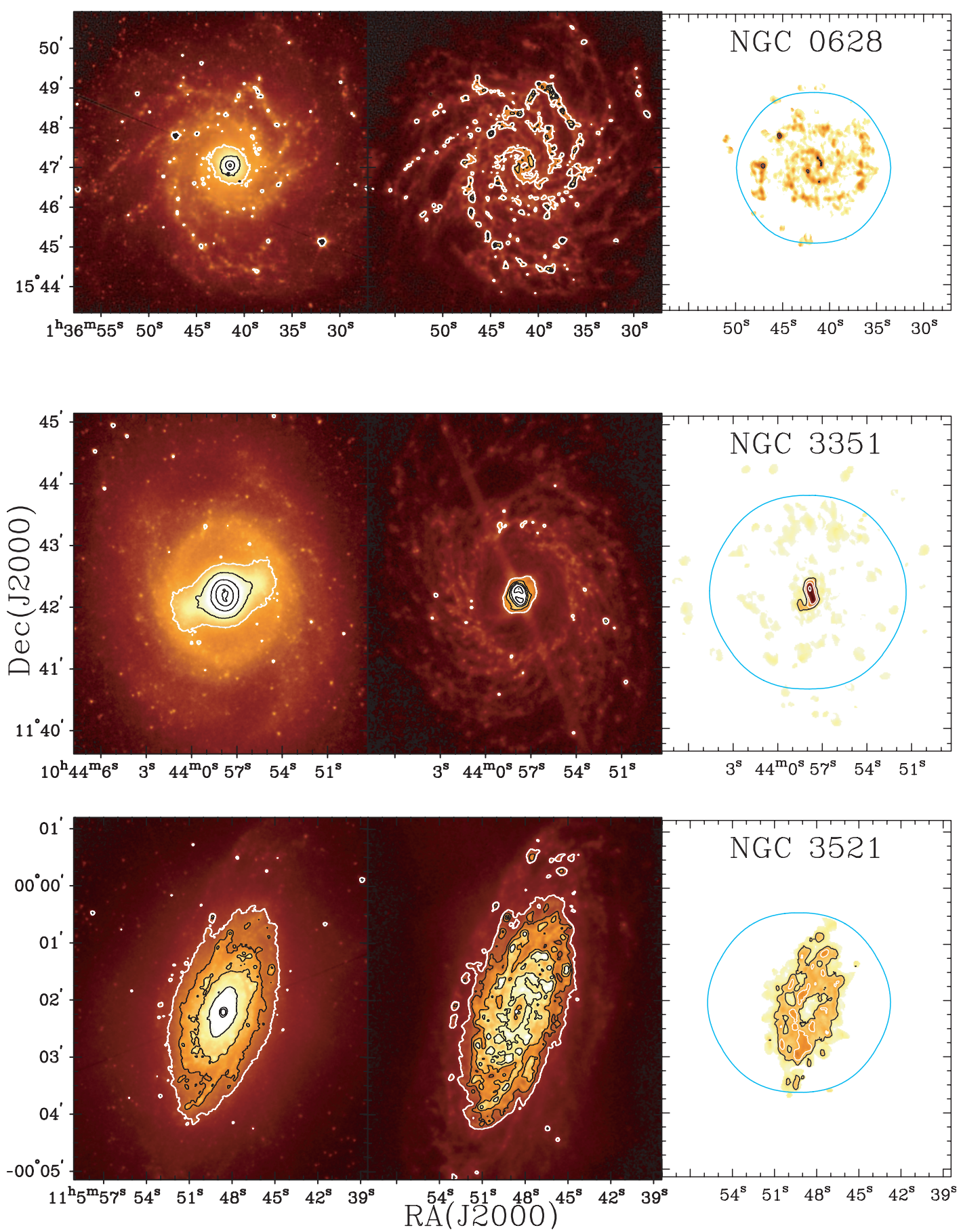

Fig. 1.-Left column: $3.6 \mu \mathrm{m}$ images of the sample galaxies. At this wavelength the emission is dominated by photospheric emission from red giant stars. The contours are at $1,2,4,8,32$, and $64 \mathrm{MJy} \mathrm{sr}^{-1}$. Middle column: Stellar continuum-subtracted $8 \mu \mathrm{m}$ images of the same galaxies, primarily tracing the emission from PAHs (see text). The contours are at 3, 6, 12, 24, 96, and $192 \mathrm{MJy} \mathrm{sr}^{-1}$. Right column: Total CO (1-0) emission from BIMA SONG (Regan et al. 2001) of the same galaxies. The cyan contour represents the effective limit of the CO mosaic (the $50 \%$ gain level). The contours are at $20,40,80$, and $160 \mathrm{~K} \mathrm{~km} \mathrm{~s}{ }^{-1}$.

binned the pixel values in several regions around each galaxy into bins with a width of $1 \mathrm{kJy} \mathrm{sr}^{-1}$. We then fitted a Gaussian distribution to this histogram and solved for the peak of the distribution. We estimated the uncertainty in the sky level from the variance in the sky levels in different regions around the galaxies (see Table 2). This variance ranged between 0.5 and $7 \mathrm{kJy} \mathrm{sr}^{-1}$ with a median of $1 \mathrm{kJy} \mathrm{sr}^{-1}$ at $3.6 \mu \mathrm{m}$, and between $8 \mathrm{kJy} \mathrm{sr}^{-1}$ and $95 \mathrm{kJy} \mathrm{sr}^{-1}$ with a median of $15 \mathrm{kJy} \mathrm{sr}^{-1}$ at $8 \mu \mathrm{m}$. The majority of this variance is actual structure in the sky due to either a gradient in the zodiacal light across the mosaic or Galactic cirrus (for NGC 6946). Note that the median sky levels are $20 \mathrm{kJy} \mathrm{sr}^{-1}$ at $3.6 \mu \mathrm{m}$ and $2.5 \mathrm{MJy} \mathrm{sr}^{-1}$ at $8 \mu \mathrm{m}$. Therefore, we are able to determine the sky level to about $5 \%$ at $3.6 \mu \mathrm{m}$ and $0.5 \%$ at $8 \mu \mathrm{m}$.

For two of the galaxies there is a large amount of structure in the sky at $8 \mu \mathrm{m}$. In NGC 628 there is a strong gradient in the sky, presumably due to zodiacal emission. We removed this gradient by creating a one-dimensional vector from a rectangular region off of the galaxy. The value of each pixel in the vector was the median of the corresponding row in the region. We then created a sky image by expanding the vector to be the same size as the 

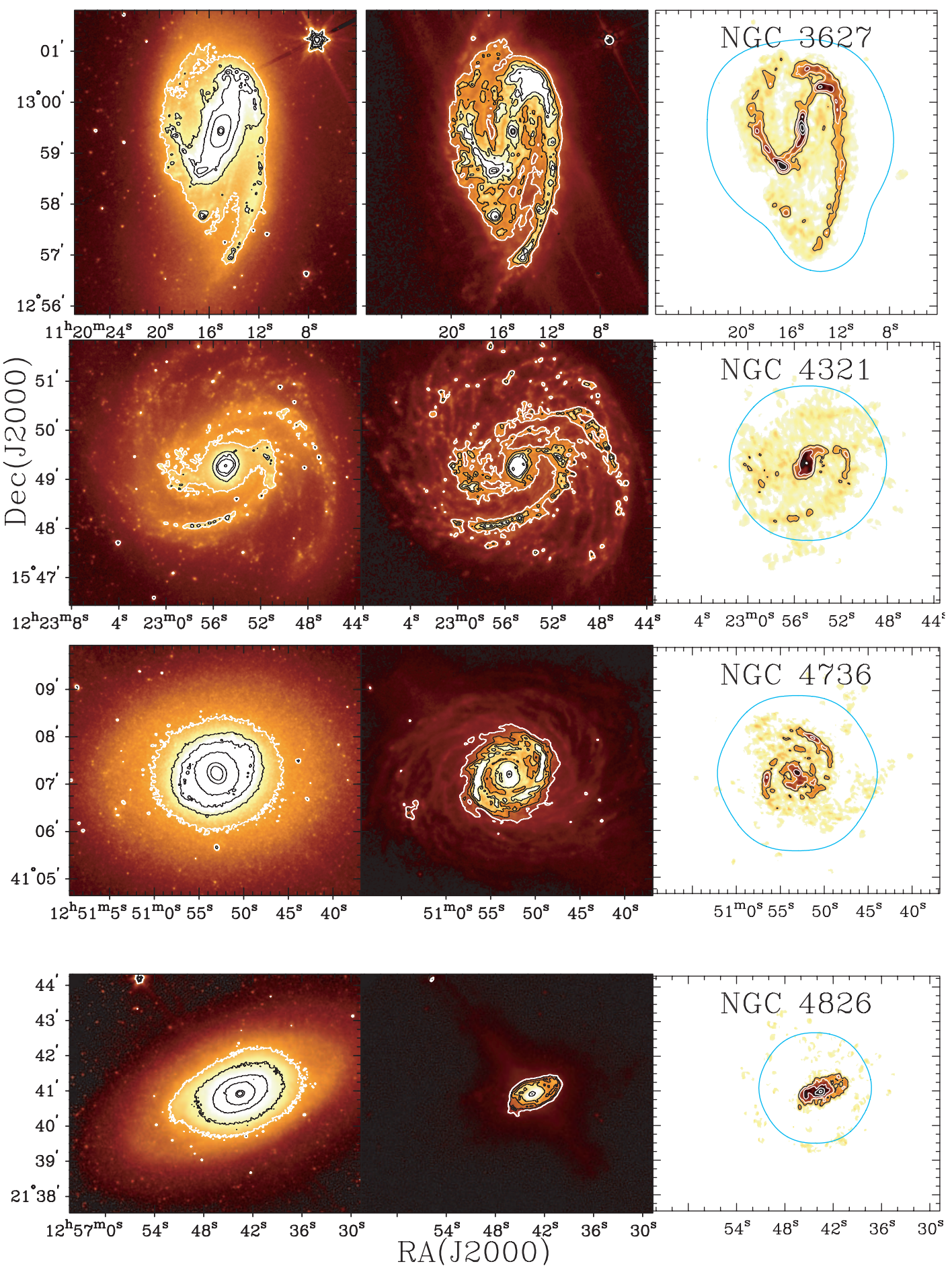

FIg. 1._Continued

original image. We then subtracted the sky image, forming a sky-subtracted image. For NGC 6946, due to its low galactic latitude, there is a lot of structure (cirrus) in the sky. The galaxy itself appears to be in a locally lower sky region, which would lead to an oversubtraction of sky level if we just used the average sky level. Therefore, we used a region just past the edge of the galaxy to find the sky level. This leads to a nonzero intensity beyond the edge of the $8 \mu \mathrm{m}$ disk.

To find the radial profiles of the surface brightness, we used the position angles and inclinations from Regan et al. (2001), which were determined either from the stellar light or from the
CO kinematics (see Table 1). We then measured the average surface brightness in concentric elliptical annuli for each source for the $8 \mu \mathrm{m}$ profiles. For the $3.6 \mu \mathrm{m}$ profiles, we used the median brightness in the annuli to filter out the effect of foreground stars. We fixed the centers for all profiles at the position of the peak in the stellar maps. For the CO profiles, we used the results of Regan et al. (2001). In Figure 2 we show the radial profiles of the $3.6 \mu \mathrm{m}$ (stars), $8 \mu \mathrm{m}$ (PAHs), and CO $1-0$ emission (molecular gas). The uncertainty in the surface brightnesses of the galaxies in the IRAC profiles is dominated by the variance in the regional sky levels. Therefore, we used the measured 

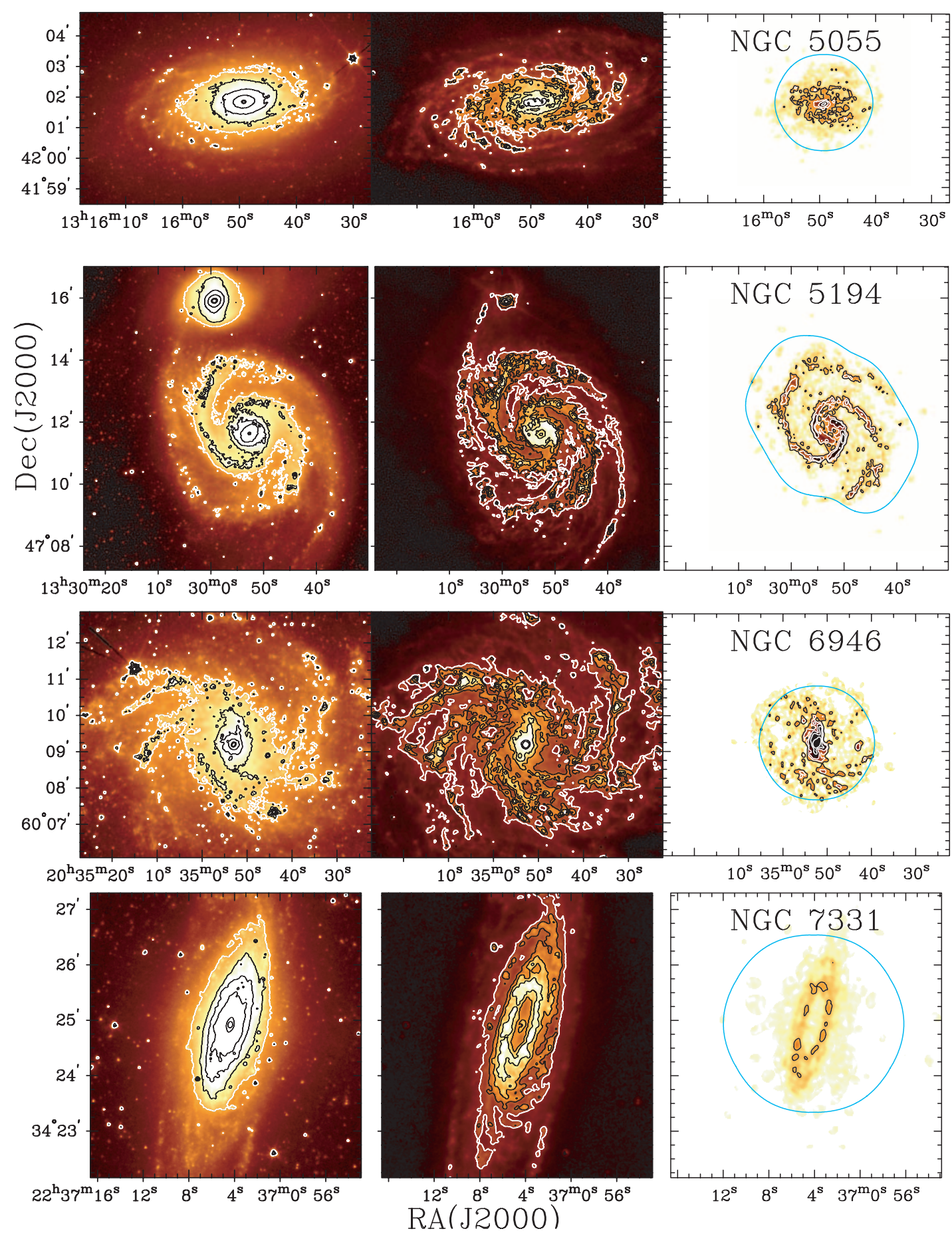

FIG. 1._Continued

variance in the regional sky levels for the error bars and used a linear spacing of annuli.

\section{RESULTS}

\subsection{Galaxy Images}

Figure 1 shows the wealth of information that is present in IRAC images of nearby spirals. As extinction of starlight is virtually absent in the mid-infrared at $3.6 \mu \mathrm{m}$, all galaxies look more symmetric than they do in optical light. The $3.6 \mu \mathrm{m}$ images show the stellar surface density through the emission from stellar photospheres, including the prominent strong bar in NGC 3351. In some places, there are significant contributions from young red supergiants at $3.6 \mu \mathrm{m}$ seen as bright, small-scale structures in an otherwise diffuse disk (e.g., the southern spiral arm of NGC 4321). At $8 \mu \mathrm{m}$ the spiral structure is more prominent than at $3.6 \mu \mathrm{m}$, and a variety of complicated local structures can be seen, e.g., supershells along the spiral arms (in NGC 628, NGC 5194 , and NGC 6946), and spurs or ridges of emission connecting the spiral arms (in NGC 5194 and NGC 6946).

Figure 1 also shows the excellent qualitative agreement between the $\mathrm{CO}$ images and the $8 \mu \mathrm{m}$ PAH images of the galaxies. 
TABLE 2

Galaxy Sensitivities

\begin{tabular}{|c|c|c|c|}
\hline Galaxy & $\begin{array}{c}\text { CO Noise Level } \\
\left(\mathrm{mK} \text { in } 10 \mathrm{~km} \mathrm{~s}^{-1} \text { channel) }\right.\end{array}$ & $\begin{array}{c}3.6 \mu \text { m Sky Variance } \\
\left(\mathrm{kJy} \mathrm{sr}^{-1}\right)\end{array}$ & $\begin{array}{c}8 \mu \mathrm{m} \text { Sky Variance } \\
\left(\mathrm{kJy} \mathrm{sr}^{-1}\right)\end{array}$ \\
\hline 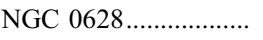 & 130 & 7 & 19 \\
\hline NGC $3351 \ldots \ldots \ldots \ldots \ldots . .$. & 160 & 6 & 27 \\
\hline NGC $3521 \ldots \ldots \ldots \ldots \ldots . .$. & 150 & 1 & 50 \\
\hline NGC $3627 \ldots \ldots \ldots \ldots . . . .$. & 100 & 2 & 15 \\
\hline NGC $4321 \ldots \ldots \ldots \ldots \ldots . .$. & 130 & 1 & 17 \\
\hline NGC 4736.................... & 170 & 4 & 7 \\
\hline NGC 4826.................... & 200 & 3 & 6 \\
\hline NGC $5055 \ldots \ldots \ldots \ldots . . . .$. & 160 & 1 & 4 \\
\hline NGC 5194................... & 190 & 1 & 10 \\
\hline 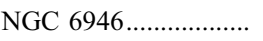 & 190 & 5 & 95 \\
\hline 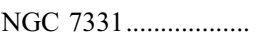 & 150 & 3 & 8 \\
\hline
\end{tabular}

Most of the differences in the images can be explained by the larger field of view, higher resolution, and higher sensitivity of the $8 \mu \mathrm{m}$ images. These PAH images provide a new window onto the ISM at sensitivities that cannot be reached by millimeter or centimeter radio observations. We can estimate the relative sensitivities of the two sets of images by comparing the noise levels in the NGC 5194 (M51) maps. M51 is a good choice because the ISM is almost completely molecular within the stellar disk of M51. In the $8 \mu \mathrm{m}$ continuum-subtracted map, we estimate the noise level at $0.1 \mathrm{MJy} \mathrm{sr}^{-1}$. Scaling by the relative flux levels in the spiral arms of the $8 \mu \mathrm{m}$ and CO images, this is approximately the equivalent of $0.3 \mathrm{Jy}$ beam ${ }^{-1} \mathrm{~km} \mathrm{~s}^{-1}$ in the
CO total intensity map. We can ignore the contribution of the atomic hydrogen because the ISM in M51 is almost completely molecular in this region. The actual noise in the BIMA SONG $\mathrm{CO}$ integrated line intensity map is around $5 \mathrm{Jy}_{\text {beam }}^{-1} \mathrm{~km} \mathrm{~s}^{-1}$. Scaling by the factor of 3 higher resolution in the $8 \mu \mathrm{m}$ map means that, at the same resolution, the $8 \mu \mathrm{m}$ map is at least 90 times more sensitive than the BIMA SONG CO map.

\subsection{Radial Profiles}

Figure 2 shows that we can detect both the stellar (at $3.6 \mu \mathrm{m}$ ) and $8 \mu \mathrm{m}$ light well beyond $R_{25}$ in many of the galaxies. In all of the galaxies we can accurately measure the average disk stellar
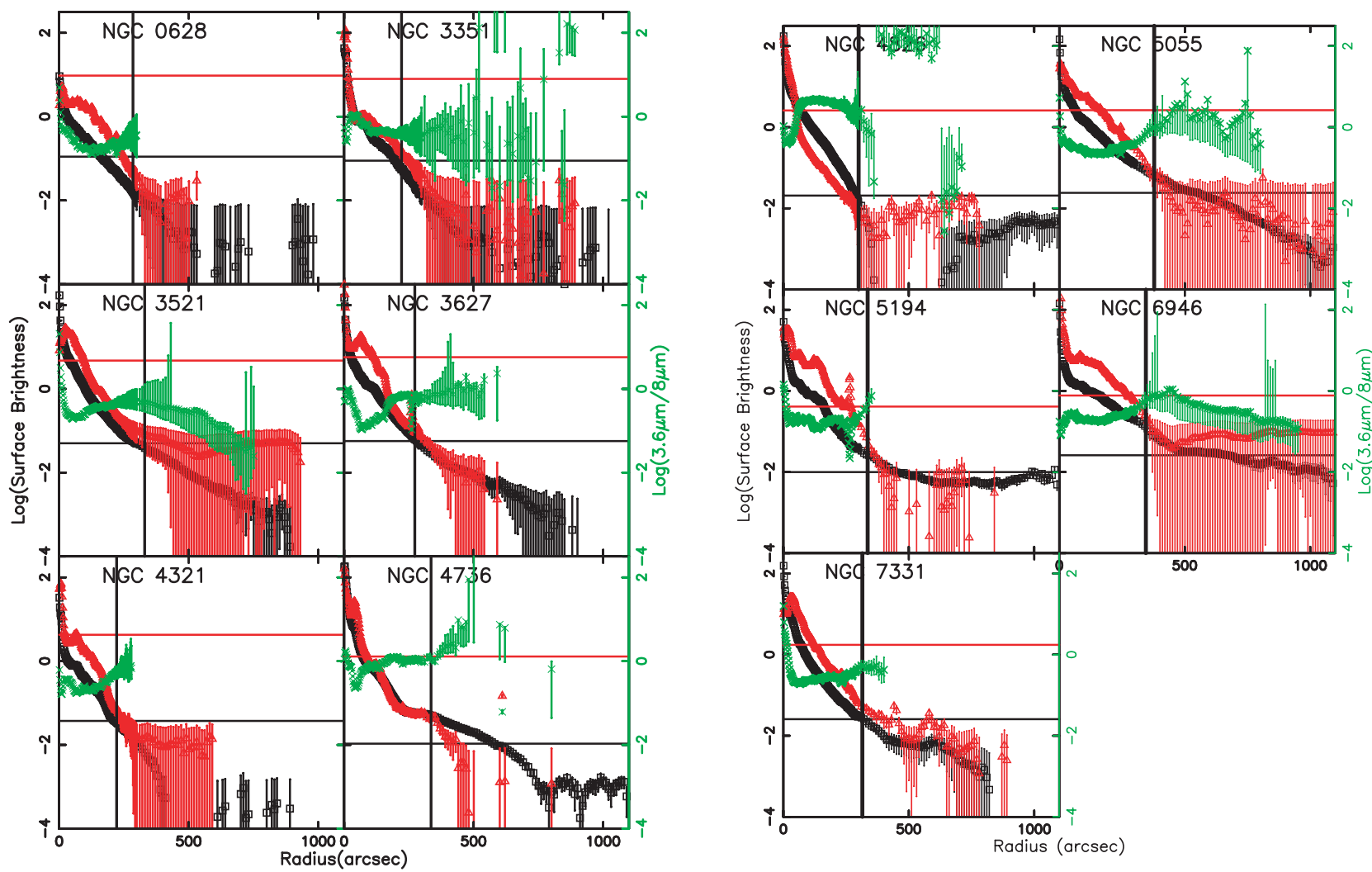

FIG. 2.- Radial profiles of the $3.6 \mu \mathrm{m}$ stellar (black squares) and $8 \mu \mathrm{m}$ PAH emission (red triangles) for the sample galaxies. In addition, the green points (marked with crosses) represent the ratio of the surface brightnesses (right scale). The surface brightness is measured in $\mathrm{MJy} \mathrm{sr}^{-1}$. The values plotted are the median for the $3.6 \mu \mathrm{m}$ emission and mean for the $8 \mu \mathrm{m}$ emission in each elliptical annulus, and the error bars are the uncertainty in the sky level. A vertical bar shows the $R_{25}$ radius for each galaxy. The horizontal bar shows the average sky level for the 3.6 and $8 \mu \mathrm{m}$ emission in each galaxy. 

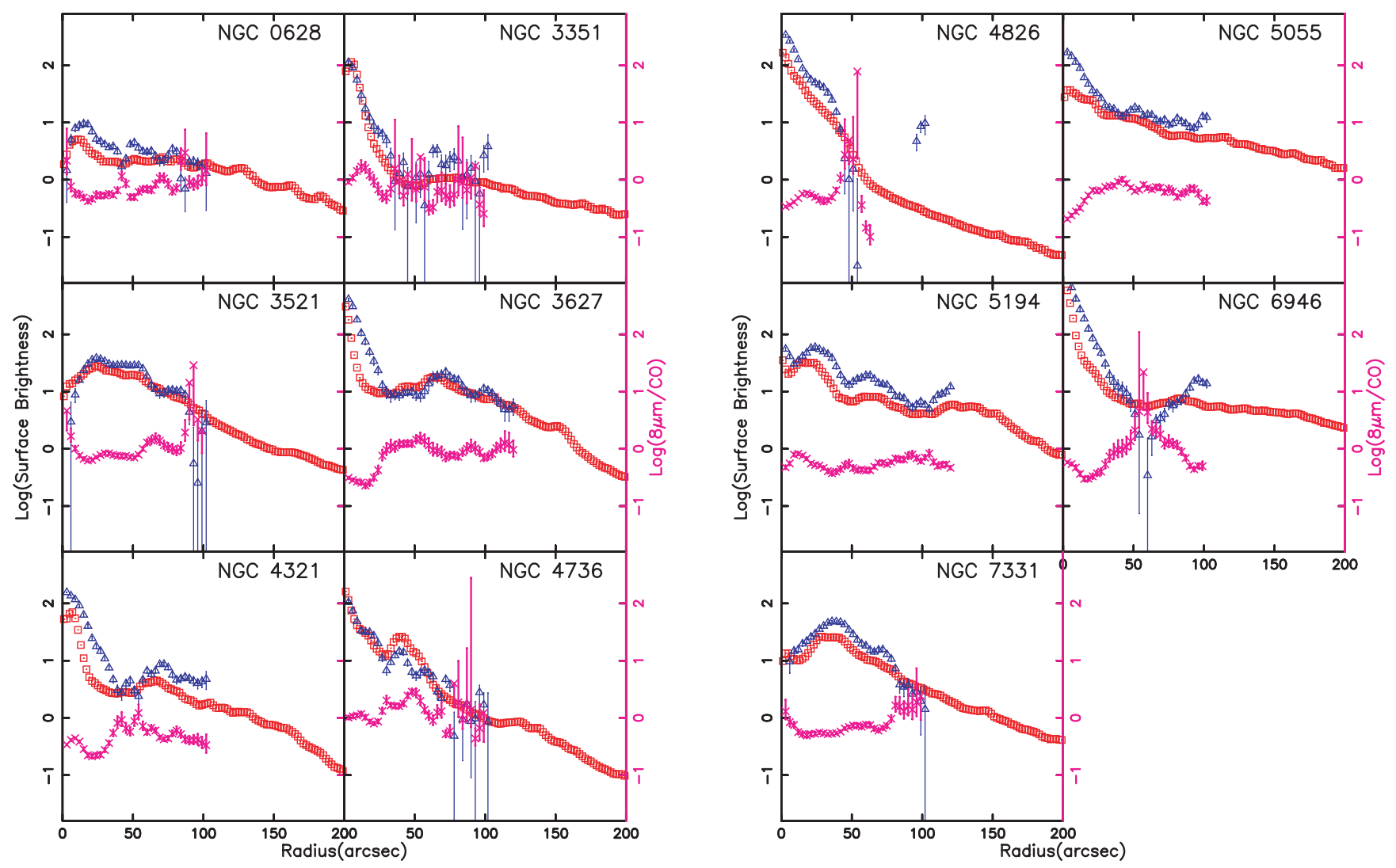

FIG. 3.-Radial profiles of the $8 \mu \mathrm{m} \mathrm{PAH} \mathrm{(red} \mathrm{squares)} \mathrm{and} \mathrm{CO} \mathrm{emission} \mathrm{(blue} \mathrm{triangles)} \mathrm{for} \mathrm{the} \mathrm{sample} \mathrm{galaxies.} \mathrm{In} \mathrm{addition,} \mathrm{the} \mathrm{magenta} \mathrm{points} \mathrm{(marked} \mathrm{as} \mathrm{crosses)}$ represent the ratio of the $8 \mu \mathrm{m}$ to the CO surface brightnesses (right scale). The $8 \mu \mathrm{m}$ surface brightness is measured in MJy sr ${ }^{-1}$, and the CO surface brightness is measured in $\mathrm{K} \mathrm{km} \mathrm{s}^{-1}$. The values plotted are the mean for the emission in each elliptical annulus, and the error bars are the uncertainty in the sky level for the $8 \mu \mathrm{m}$ emission and the rms of the mean in each annulus for the $\mathrm{CO}$ emission.

surface brightness to a radius of at least $1.5 R_{25}$, and in some galaxies we can detect it beyond $2 R_{25}$. In most of the galaxies, the stellar surface brightness varies smoothly out to the radius at which the detection limit is reached. In only two galaxies, NGC 4321 and NGC 4826, do we see sharp changes in the slope and a possible edge to the stellar disks, but we cannot rule out the possibility that this may be an artifact of background oversubtraction.

Our $3.6 \mu \mathrm{m}$ stellar profiles extend out to radii and surface brightness levels at which we can detect deviations from exponential profiles. In NGC 4736, there is an outer stellar ring that dominates the surface brightness at $R>350^{\prime \prime}$ (Waller et al. 2001 and references therein). In NGC 5194, beyond $R>500^{\prime \prime}$, we are detecting the diffuse stellar light from the interaction with NGC 5195. This light has a constant surface brightness and extends well beyond the disk of NGC 5194. In NGC 6946 we see a break in the surface brightness profile at around $500^{\prime \prime}$, where the scale length seems to change.

Our excellent $3.6 \mu \mathrm{m}$ surface brightness sensitivity $\left(0.5 \mathrm{kJy} \mathrm{sr}^{-1}\right.$ for the best images) allows us to trace the stellar exponential disks in the galaxies over a significant number of scale lengths. In NGC 3521, NGC 3627, and NGC 5055 we detect light from the disk over a factor of 6000-9000 in surface brightness, or about 89 disk scale lengths.

The $8 \mu \mathrm{m}$ PAH radial profiles often have similar slopes to the $3.6 \mu \mathrm{m}$ stellar profiles in the inner disks of the galaxies. Beyond $R_{25}$ the uncertainties in the background levels for many of the galaxies make it impossible to determine the shape of the $8 \mu \mathrm{m}$ emission profile. The flat surface brightness profiles at large radii we see in NGC 3521 and NGC 6946 may be due to Galactic cirrus emission, but there are also uncertainties in the sky level. So it could just be a background subtraction error. The largest radius at which we can clearly see an exponential $8 \mu \mathrm{m}$ profile is in NGC 3627, where the light profile is exponential to a radius of at least $13 \mathrm{kpc}\left(500^{\prime \prime}\right)$.

There is a tendency for $I_{\nu}(8 \mu \mathrm{m}$ PAH $)>I_{\nu}(3.6 \mu \mathrm{m})$. The median value of $I_{\nu}(8 \mu \mathrm{m} \mathrm{PAH}) / I_{\nu}(3.6 \mu \mathrm{m})$ is approximately 2 , with a range of $1-4$. This is true for all galaxies except for NGC 4826 , in which $I_{\nu}(3.6 \mu \mathrm{m} \mathrm{PAH})>I_{\nu}(8 \mu \mathrm{m})$ within the disk $\left(50^{\prime \prime}<r<300^{\prime \prime}\right)$, and NGC 4736, in which the surface brightness at 3.6 and $8 \mu \mathrm{m}$ is similar. For those galaxies in which the $8 \mu \mathrm{m}$ emission is brighter than the $3.6 \mu \mathrm{m}$ emission, the ratio of their surface brightnesses is similar in all of the galaxies.

In Figure 3 we plot the $8 \mu \mathrm{m}$ and $\mathrm{CO}$ surface brightness radial profiles and their ratio. There is general agreement between the radial profiles of the $\mathrm{CO}$ emission and the $8 \mu \mathrm{m}$ PAH emission. Qualitatively, they not only have the same general scale length, but most of the deviations from a simple exponential disk are similar. This is not unexpected, given the strong correlation between the $\mathrm{CO}$ and $8 \mu \mathrm{m}$ images that we see in Figure 1. In addition, there is not a large variation in the ratio of their surface brightnesses (as long as both tracers are detected at high signal-to-noise ratio), except in the central regions of the galaxies NGC 3627, NGC 5055, NGC 4321, and NGC 6946. (Throughout this paper, we use the term "central region" to denote the area of enhanced $\mathrm{CO}$ emission above the exponential profile.) For a significant range 
TABLE 3

Disk Scale Lengths

\begin{tabular}{|c|c|c|c|}
\hline Galaxy & $\begin{array}{l}\text { Stellar Scale Length } \\
\qquad(\mathrm{kpc})\end{array}$ & $\begin{array}{c}8 \mu \mathrm{m} \text { Scale Length } \\
(\mathrm{kpc})\end{array}$ & $\begin{array}{c}\text { CO Scale Length } \\
(\mathrm{kpc})\end{array}$ \\
\hline NGC $0628 \ldots \ldots \ldots \ldots . . . . . . . . .$. & $3.0 \pm 0.3$ & $3.4 \pm 0.3$ & $5.8 \pm 0.2$ \\
\hline NGC $3351 \ldots \ldots \ldots \ldots . . . . . . .$. & $3.0 \pm 0.3$ & $4.1 \pm 0.4$ & $2.6 \pm 0.3$ \\
\hline NGC $3521 \ldots \ldots \ldots \ldots . . . . . . . .$. & $1.7 \pm 0.2$ & $1.6 \pm 0.2$ & $2.3 \pm 0.1$ \\
\hline 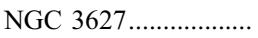 & $3.3 \pm 0.3$ & $2.9 \pm 0.3$ & $1.8 \pm 0.6$ \\
\hline NGC $4321 \ldots \ldots \ldots \ldots . . . . . . .$. & $4.6 \pm 0.5$ & $3.9 \pm 0.4$ & $2.8 \pm 0.3$ \\
\hline NGC $4736 \ldots \ldots \ldots \ldots . . . . . . . .$. & $1.2 \pm 0.1$ & $1.0 \pm 0.1$ & $1.0 \pm 0.1$ \\
\hline NGC $4826 \ldots \ldots \ldots \ldots . . . . . . . .$. & $1.8 \pm 0.2$ & $1.7 \pm 0.2$ & $1.2 \pm 0.2$ \\
\hline NGC $5055 \ldots \ldots \ldots \ldots \ldots . . . . . .$. & $1.6 \pm 0.2$ & $1.4 \pm 0.1$ & $2.6 \pm 0.1$ \\
\hline NGC $5194 \ldots \ldots \ldots \ldots \ldots . . . . . . . .$. & $1.8 \pm 0.2$ & $1.7 \pm 0.2$ & $2.8 \pm 0.1$ \\
\hline NGC $6946 \ldots \ldots \ldots \ldots \ldots . . . . . . .$. & $4.6 \pm 0.5$ & $3.8 \pm 0.4$ & $2.1 \pm 0.3$ \\
\hline NGC $7331 \ldots \ldots \ldots \ldots . . . . . . .$. & $3.6 \pm 0.4$ & $3.3 \pm 0.3$ & $5.6 \pm 0.4$ \\
\hline
\end{tabular}

in radii in each of the galaxies, there is an approximately linear relationship between $\mathrm{CO}$ emission and $8 \mu \mathrm{m}$ PAH emission, with $I_{\mathrm{CO}} \approx 2 \mathrm{~K} \mathrm{~km} \mathrm{~s}^{-1} I_{\nu}(8 \mu \mathrm{m} \mathrm{PAH}) / \mathrm{MJy} \mathrm{sr}^{-1}$.

In the central regions of the galaxies, where the $\mathrm{CO}$ and $8 \mu \mathrm{m}$ do not have the same ratio, the $\mathrm{CO}$ emission is brighter than the $8 \mu \mathrm{m}$ emission. This effect is strong enough in NGC 5055 that the central excess of $\mathrm{CO}$ emission is not seen in the $8 \mu \mathrm{m}$ emission. In NGC 3627 the central excess is seen in both the CO and $8 \mu \mathrm{m}$ emission, but is weaker in the $8 \mu \mathrm{m}$ emission.

When we look qualitatively we see that the PAH scale lengths in most of the galaxies are close to, but slightly smaller than, the stellar light. The similarity in scale lengths has been seen before in CO observations (Young \& Scoville 1982; Regan et al. 2001) and in ISO dust observations of NGC 6946 (Malhotra et al. 1996).

In 6 of the 11 galaxies (NGC 3351, NGC 3627, NGC 4321, NGC 4736, NGC 4826, and NGC 6946) there is a central component of the $8 \mu \mathrm{m}$ PAH emission that is brighter than the inward extrapolation of the exponential disk. Note that the exponential disk is not well defined in NGC 4736 and NGC 4826, but there is a central excess in both the $\mathrm{CO}$ and $8 \mu \mathrm{m}$ in both of these galaxies (Regan et al. [2001] did not claim that NGC 4736 had a central CO excess, but the excess is similar to the one in NGC 4826). In the remaining five galaxies, four galaxies show no central excess in either PAH or CO emission. Only one galaxy in this sample, NGC 5055, exhibits an excess in CO emission, which is not echoed in $8 \mu \mathrm{m}$ PAH emission.

\section{DISCUSSION}

\subsection{The Relationship between the Stellar, CO, and PAH Profiles}

By measuring the shapes of radial profiles in $3.6 \mu \mathrm{m}, 8 \mu \mathrm{m}$, and $\mathrm{CO}$ emission, we can further quantify the similarities seen in the images of each galaxy in different tracers. To measure the disk scale lengths we fitted exponential radial profiles to the light profiles outside the central regions to avoid both the depressions and excesses in the light profiles in the central regions. Table 3 shows the measured scale lengths. In Figure 4 we plot the scale lengths of the PAH $(8 \mu \mathrm{m}), \mathrm{CO}$, and stellar $(3.6 \mu \mathrm{m})$ disks against one another. There is clearly a strong correlation between them all, with the best correlation being between the stellar and PAH scale lengths.

The scale lengths of the stellar light, $\mathrm{CO}$ emission, and PAH emission in the 11 galaxies are remarkably similar. The PAH profiles are a better match to the stellar profiles than are the $\mathrm{CO}$ profiles. In most cases, the PAH emission has a slightly smaller scale length than the stellar emission. The two galaxies with larger PAH scale lengths than $\mathrm{CO}$ scale lengths are NGC 3351 and NGC 4736. In NGC 3351 the larger PAH scale length is evident from the profile. In NGC 4736 neither the stellar light nor the PAH emission is well fitted by an exponential disk. We also detect PAH emission at large radii in this galaxy.

There are a number of possible reasons for the differences between the $\mathrm{CO}$ and PAH radial profiles represented in Figure 3. The CO profile may be affected by residual sidelobes that are an observational artifact from bright sources (e.g., NGC 6946). There is also the debate about the $\mathrm{CO}$ emissivity that may lead to an enhancement of CO emission (Regan 2000). And finally, we note that PAH emission may be present even when $\mathrm{CO}$ emission is weak, e.g., in outer regions of galaxies, where the atomic phase of the ISM begins to dominate.

These observations are more evidence for the molecular ISM in galaxies having an exponential profile. Most early CO singledish observations did not map the disk of the galaxy, but instead observed several positions along the major axis of the disk (e.g., Young \& Scoville 1982; Sage 1993a, 1993b; Young et al. 1995). While these observations are consistent with the molecular ISM being distributed in an exponential disk, they do not strongly constrain the functional form of the distribution. Our observations have much higher spatial resolution than the single-dish CO observations, and higher sensitivity than the interferometric $\mathrm{CO}$ observations. By extending the detection of the molecular ISM to large radii, we are able to better constrain the functional form of the distribution. In Figure 2, we see that the PAH profile is best described as a straight line (in this log-linear plot) over the majority of the disk, implying that the ISM is distributed in an exponential disk. Because the $8 \mu \mathrm{m}$ PAH emission only traces the ISM indirectly, due to its relationship to the stars formed from the ISM, this is not a direct measurement of the exponential radial profile of the ISM surface brightness.

\subsection{The Influences on PAH Emission}

PAH emission requires that there be optical and /or UV photons (Leger \& Puget 1984; Mattioda et al. 2005). In other words, the PAH emission should be proportional to both the number of exciting photons and the number of PAH particles. Why then is the PAH flux proportional to the amount of ISM material (the CO flux)?

One place where we might expect to see a deviation between the PAH and $\mathrm{CO}$ emission is near star-forming regions, in which the PAH emission should increase due to the higher radiation field, or decrease if the PAHs are destroyed. In these regions, the 


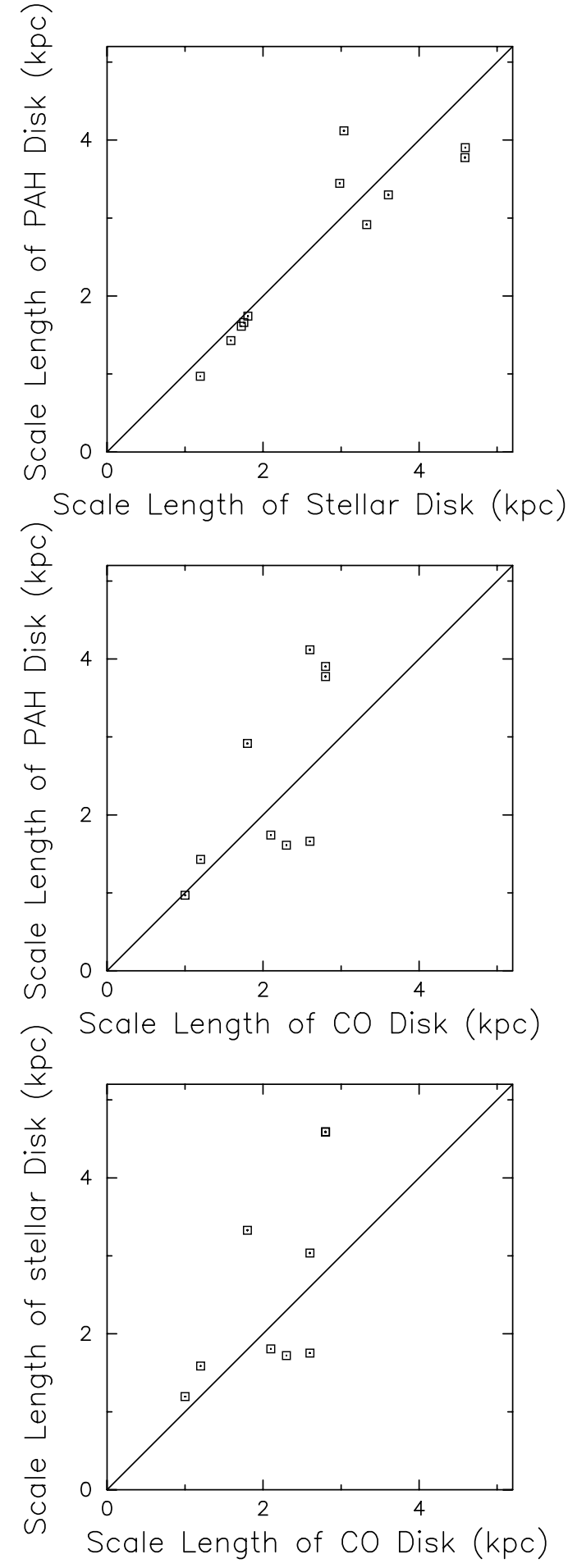

FIG. 4.-Top panel: Scale lengths of the stellar and PAH disks for each galaxy. Middle panel: Scale lengths of the $\mathrm{CO}$ and PAH disks for each galaxy. Bottom panel: Scale lengths of the $\mathrm{CO}$ and stellar disks for each galaxy.

molecular gas emission is likely to be higher because stars form in molecular clouds. The increase in the $\mathrm{CO}$ emission could be echoed by an increase in the PAH emission if the ionizing photons are absorbed by the gas, whereas a substantial fraction of the nonionizing $(h \nu<13.6 \mathrm{eV})$ photons are absorbed by dust. If the star formation rate were approximately proportional to the $\mathrm{CO}$ intensity, and an approximately constant fraction of photons radiated by $\mathrm{O}$ and $\mathrm{B}$ stars were absorbed by PAHs, then the $\mathrm{PAH}$ emission would be approximately proportional to the $\mathrm{CO}$ emis- sion. If instead of photons from star-forming regions, PAH emission is primarily excited by ambient galactic stellar flux, then the PAH emission should vary with the number of PAH particles instead of the local star light intensity.

Our observations of the PAH morphology relative to the stellar morphology provide some evidence that the mean path length of the exciting photons is large. If the mean path length were short, then regions of high stellar surface brightness should produce more PAH flux from each PAH particle, and one would expect that the PAH flux would be higher relative to the CO flux in the central regions of disk galaxies. Instead, some of the largest deviations from a constant ratio of surface brightnesses are in the central regions of NGC 3627, NGC 4321, and NGC 5055, where there is less $\mathrm{PAH}$ emission relative to the $\mathrm{CO}$ emission. Furthermore, the suggestions of an increasing ratio of PAH emission relative to stellar emission in the outer disks of some of the galaxies (NGC 3521 and NGC 4321) imply that the PAH emission cannot be a measure of the locally generated photons. Note that the uncertainty in the sky subtraction limits our claim that the outer $8 \mu \mathrm{m}$ surface brightness profiles are flat in NGC 3521 and NGC 4321.

The critical point for this paper is that the ratio of the PAH emission to the $\mathrm{CO}$ emission is not wildly variable over the optical disk. This means that, to first order, one can use the PAH radial profile as a proxy for the molecular ISM mass density radial distribution within the optical disks of the galaxy.

\subsection{Bars, Mass Inflow, and Secular Evolution}

The PAH profiles provide further evidence that bars are inducing a mass inflow in these galaxies. The four galaxies with the largest excesses of PAH emission in their central region are all barred (NGC 3351, NGC 3627, NGC 4321, and NGC 6946). We can see from their $3.6 \mu \mathrm{m}$ images in Figure 1 that NGC 3351 and NGC 3627 have large-scale prominent galactic bars. NGC 4321 and NGC 6946 have weaker bars. The correlation of apparent bar strength with excess central ISM content implies that the largescale bars are driving the gas to smaller radii, a conclusion consistent with the CO results of Sakamoto et al. (1999) and Sheth et al. (2005). This is also consistent with both $\mathrm{H} \alpha$ (Regan et al. 1997; Zurita et al. 2004) and CO observations (Regan et al. 1999; Sheth et al. 2000, 2002) of the inflowing gas in barred galaxies.

All of the galaxies with central holes in their PAH distributions are unbarred galaxies. We see central holes in the radial PAH distributions in NGC 628, NGC 3521, and NGC 7331. NGC 628 and NGC 7331 are clearly unbarred galaxies both in their RSA classification (Sandage \& Tammann 1981) and from their $3.6 \mu \mathrm{m}$ images (Fig. 1). Although NGC 3521 is classified as SAB in the RSA, we do not see any signs of a bar or oval distortion in the $3.6 \mu$ m image; therefore, NGC 3521 is not counted as a barred galaxy for this work.

Without continuous radial inflow of gas, one would expect the center of an exponential disk to use up its fuel faster than regions of lower surface density. If the star formation rate per unit area $S$ is related to the surface density of gas $\Sigma$ by a relation of the form $S \propto \Sigma^{1.4}$ (Kennicutt 1998), then higher density regions will be depleted of gas faster than lower density regions. This could explain why some of the unbarred galaxies have central holes in their ISM distributions, similar to some of the strongly barred, early-type galaxies in Sheth et al. (2005).

The central excesses that we see in the PAH radial profiles confirm the results of Regan et al. (2001), Sheth et al. (2005), and Sakamoto et al. (1999), and make it unlikely that the excess $\mathrm{CO}$ emission is caused, generally, by an increase in the amount of the $\mathrm{CO}$ emission per $\mathrm{H}_{2}$ molecule (one possible exception in this 
sample: NGC 5055). The PAH emission arises from a completely separate process, and although it is possible that the differences in the conditions in the central regions of galaxies could vary to increase both the $\mathrm{CO}$ and $\mathrm{PAH}$ emission per unit $\mathrm{H}_{2}$ molecule, it would be fortuitous if they increased by the same amount.

Another interesting characteristic of the radial profiles is the relatively constant relationship between the surface brightness of the PAH emission and that of the stellar emission for 9 of the 11 galaxies (NGC 4736 and NGC 4826 are exceptions). To quantify this, for each galaxy we measured the total $8 \mu \mathrm{m} \mathrm{PAH}$ to the $3.6 \mu \mathrm{m}$ surface fluxes inside of $R_{25}$ for each galaxy. The median of these 11 average ratios is 3 . Therefore, the intensity $I_{\nu}$ in IRAC band 4 (dominated by PAH emission) is approximately 3 times the specific intensity near $3.6 \mu \mathrm{m}$ due to stellar emission.

Regan et al. (2001) saw a similar but weaker relationship between the surface brightnesses of the stellar and CO distributions. A relationship between the stellar and ISM surface densities over a variety of galaxies implies that there must be a feedback mechanism. Star formation is the logical mechanism because it transforms ISM surface density into stellar surface density (Kennicutt 1989). One way the two could be connected is that if, over time, the star formation at a given radius is proportional to the ISM surface density; the amount of stars at that radius would be proportional to the average ISM surface density at that radius. This implies that the star formation rate at a given radius is relatively constant over the life of the stellar disk. Alternatively, a faster feedback mechanism could result if the ISM surface density exceeds some equilibrium value; the rate of star formation would increase, lowering the ISM surface density to the equilibrium value. Conversely, if the ISM surface density is below the equilibrium value, star formation will decrease or even stop until, through a process such as inflow from larger radii, the ISM surface density increases high enough to allow star formation to occur. If this equilibrium value was proportional to the stellar surface density, there would be a relationship between the ISM and stellar surface densities.

What is striking about the relationship between the stellar and PAH median radial surface brightnesses is that at a given radius the two surface brightnesses show a large variation. For example, the PAH emission is much more concentrated in the spiral arms than the $3.6 \mu \mathrm{m}$ emission (see Fig. 1). This concentration is what one would expect, because the ISM is dynamically cold and responds more strongly to perturbations in the potential. Yet, when we average over all azimuth angles, the ratio of the surface brightnesses does not vary greatly. This implies a long timescale for the feedback so that the star formation in the spiral arms can average out as the spiral density wave rotates through the disk.

\section{CONCLUSIONS}

We have shown that the radial profiles of the $8 \mu \mathrm{m}$ PAH emission in a set of spiral galaxies are a good match to the radial profiles of their $\mathrm{CO}$ emission. In all but 1 of the 11 galaxies we examined, the central excess or depression seen in the $\mathrm{CO}$ emission is also seen in $8 \mu \mathrm{m}$ emission. The detection of central excesses in PAH emission in 6 out of 7 galaxies observed to have $\mathrm{CO}$ excesses suggests that it is unlikely that the $\mathrm{CO}$ excess is due generally to a variation in the relationship between $\mathrm{CO}$ flux and molecular gas mass. Excesses are expected in the centers of galaxies if the gas is flowing inward as part of the secular evolution of the galaxy.

We saw a central excess of $8 \mu \mathrm{m}$ PAH emission in all four of the barred galaxies in the sample. This is consistent with both previous CO studies (Sakamoto et al. 1999; Sheth et al. 2005) that have shown this kind of excess, and kinematic observations of inflowing ionized (Regan et al. 1997; Zurita et al. 2004) and molecular gas (Regan et al. 1999).

Our 3.6 $\mu \mathrm{m}$ photometry is able to detect the exponential disks of several galaxies in the sample out to nine disk scale lengths.

We also find a relatively constant relationship between the stellar and PAH surface brightnesses for the majority of the galaxies. This relationship between the two implies that there is a feedback mechanism between the stellar surface density and the ISM surface density.

Finally, we find a reasonably constant ratio between the $\mathrm{CO}$ and $8 \mu \mathrm{m}$ PAH surface brightness in all of the galaxies in our sample. This implies that $8 \mu \mathrm{m}$ emission, despite its complicated physics, can be used as a rough tracer of the ISM in a galaxy.

It is a pleasure to acknowledge our SSC liaison scientist, Nancy Silbermann, for her capable assistance throughout the planning and early science phases of the SINGS project. Support for this work, part of the Spitzer Space Telescope Legacy Science Program, was provided by NASA through contract [1224769] issued by the Jet Propulsion Laboratory, California Institute of Technology, under NASA contract 1407.
Fazio, G. G., et al. 2004, ApJS, 154, 10

Ferrarese, L. et. al. 1996, ApJ, 464, 568

Fruchter, A. S., \& Hook, R. N. 2002, PASP, 114, 144

Graham, J. A., et al. 1997, ApJ, 477, 535

Haas, M., Klaas, U., \& Bianchi, S. 2002, A\&A, 385, L23

Helfer, T. T., Thornley, M. D., Regan, M. W., Wong, T., Sheth, K., Vogel, S. N., Blitz, L., \& Bock, D. C.-J. 2003, ApJS, 145, 259

Helou, G., Lu, N. Y., Werner, M. W., Malhotra, S., \& Silbermann, N. 2000, ApJ, 532, L21

Helou, G., et al. 2004, ApJS, 154, 253

Hughes, S. M. G., et al. 1998, ApJ, 501, 32

Kennicutt, R. C. 1989, ApJ, 344, 685 . 1998, ApJ, 498, 541

Kennicutt, R. C., et al. 2003, PASP, 115, 928

Kormendy, J., \& Kennicutt, R. C. 2004, ARA\&A, 42, 603

Leger, A., \& Puget, J. L. 1984, A\&A, 137, L5

Malhotra, S., et al. 1996, A\&A, 315, L161

Mattioda, A. L., Allamandola, L. J., \& Hudgins, D. M. 2005, ApJ, 629, 1183

Pahre, M. A., Ashby, M. L. N., Fazio, G. G., \& Willner, S. P. 2004, ApJS, 154, 235

Regan, M. W. 2000, ApJ, 541, 142

Regan, M. W., Sheth, K., \& Vogel, S. N. 1999, ApJ, 526, 97

Regan, M. W., Thornley, M. D., Helfer, T. T., Sheth, K., Wong, T., Vogel, S. N., Blitz, L., \& Bock, D. C.-J. 2001, ApJ, 561, 218

\section{REFERENCES}

Regan, M. W., Vogel, S. N., \& Teuben, P. J. 1997, ApJ, 482, L143

Regan, M. W., et al. 2004, ApJS, 154, 204

Roussel, H., Sauvage, M., Vigroux, L., \& Bosma, A. 2001, A\&A, 372, 427

Sage, L. J. 1993a, A\&A, 272, 123

1993b, A\&AS, 100, 537

Saha, A., Sandage, A., Tammann, G. A., Labhardt, L., Macchetto, F. D., \& Panagia, N. 1999, ApJ, 522, 802

Sakamoto, K., Okumura, S. K., Ishizuki, S., \& Scoville, N. Z. 1999, ApJ, 525, 691

Sandage, A., \& Tammann, G. A. 1981, Revised Shapley-Ames Catalog of Bright Galaxies (Washington: Carnegie Inst. Washington)

Sheth, K., Regan, M. W., Vogel, S. N., \& Teuben, P. J. 2000, ApJ, 532, 221

Sheth, K., Vogel, S. N., Regan, M. W., Teuben, P. J., Harris, A. I., \& Thornley, M. D. 2002, AJ, 124, 2581

Sheth, K., Vogel, S. N., Regan, M. W., Thornley, M. D., \& Teuben, P. J. 2005, ApJ, 632, 217

Tully, R. B. 1988, Nearby Galaxies Catalog (Cambridge: Cambridge Univ. Press) Waller, W. H., et al. 2001, AJ, 121, 1395

Young, J. S., \& Scoville, N. 1982, ApJ, 260, L41

Young, J. S., et al. 1995, ApJS, 98, 219

Zurita, A., Relaño, M., Beckman, J. E., \& Knapen, J. H. 2004, A\&A, 413, 73 